\title{
Optimismo disposicional, sintomatologia psicopatológica, bem-estar e rendimento académico em estudantes do primeiro ano do ensino superior ${ }^{1}$
}

\author{
Sara Otilia Marques Monteiro \\ José Pereira da Costa Tavares \\ Anabela Maria de Sousa Pereira \\ Universidade de Aveiro (Portugal)
}

\begin{abstract}
Resumo
O presente estudo ex post facto investiga a relação entre o optimismo disposicional e a sintomatologia psicopatológica, o bem-estar e o rendimento académico numa amostra de estudantes que frequentam pela primeira vez o ensino superior. Um total de 316 estudantes de uma universidade portuguesa preencheu o Life Orientation Test - Revised, o Brief Symptom Inventory, a Échelle de Mesure des Manifestations du Bien-Être Psychologique e forneceu indicadores de rendimento académico. Dos resultados obtidos, destacamos: (a) a existência de uma correlação negativa e significativa entre o optimismo disposicional e a sintomatologia psicopatológica; (b) a existência de uma correlação positiva e significativa entre o optimismo disposicional e o bem-estar; e, (c) a existência de uma correlação positiva e significativa entre o optimismo disposicional e o rendimento académico. A interpretação dos resultados, bem como as possíveis implicações destes, são discutidos à luz de literatura relevante.
\end{abstract}

Palavras-chave: optimismo disposicional; sintomatologia psicopatológica; bem-estar; rendimento académico

\begin{abstract}
Dispositional optimism, psychopathological symptomatology, well-being and academic performance among first year higher education students. The present ex post facto study investigates the relationship between dispositional optimism and psychopathological symptomatology, well-being and academic performance among first year higher education students. A total of 316 students of a Portuguese university completed the Life Orientation Test Revised, Brief Symptom Inventory, Échelle de Mesure des Manifestations du Bien-Être Psychologique and gave indicators of academic performance. From the results, we can distinguish: (a) the negative and significant correlation between dispositional optimism and psychopathological symptomatology; (b) the positive and significant correlation between dispositional optimism and well-being; and, (c) the positive and significant correlation between dispositional optimism and academic success. Interpretation of the results and their implications for student counselling are discussed in the light of the relevant literature.
\end{abstract}

Keywords: dispositional optimism; psychopathological symptomatology; well-being; academic performance

A transição para o ensino superior implica para a maioria dos jovens estudantes grandes mudanças e adaptações a novos papéis, responsabilidades e rotinas. Como qualquer período de transição de vida, trata-se de uma fase que requer a adopção de novas atitudes e comportamentos, não só no que respeita às competências de estudo, mas também à organização e autonomia do próprio aluno (Ferraz \& Pereira, 2002; Machado \& Almeida, 2001).

A investigação sugere que cerca de $40 \%$ dos estudantes experimentam problemas significativos na adaptação à universidade, conduzindo ao abandono dos estudos antes de completar a licenciatura (McLaughlin, Brozovsky, \&
McLaughlin, 1998; Pantages \& Creedon, 1978; Tinto, 1993) e ainda que, mesmo os estudantes que obtêm maior sucesso académico, podem experimentar elevados níveis de stress (Zitzow, 1984).

Em Portugal, os estudos teóricos e empíricos sobre a transição e adaptação dos estudantes do ensino superior e em particular dos estudantes do primeiro ano, apesar de serem ainda escassos, têm vindo a aumentar exponencialmente (Almeida, 2001; Diniz \& Almeida, 2005, 2006; Fernandes et al., 2005; Nico, 2000; Santos \& Almeida, 2001; Soares, 2003; Tavares, Santiago, \& Lencastre, 1998). Também no Brasil, em virtude do aumento da população estudantil e da sua heterogeneidade 
crescente, tem sido cada vez mais notória a preocupação de pesquisadores, agências formadoras e governamentais acerca da integração do estudante na vida universitária e especificamente acerca do seu desempenho académico e situação de permanência ou evasão (Vendramini et al., 2004). Neste sentido, têm sido conduzidos estudos objectivando o aumento da compreensão do processo de integração do estudante na vida universitária em contexto brasileiro (Cunha \& Carrilho, 2005; Mercuri \& Polydoro, 2003; Polydoro, Primi, Serpa, Zaroni, \& Pombal, 2001; Schleich, Polydoro \& Santos, 2006; Vendramini et al., 2004).

Os estudos realizados chamam a atenção para a necessidade de compreender a variabilidade na qualidade da adaptação demonstrada pelos estudantes aos desafios e exigências que lhes são colocados, como um processo multideterminado e complexo nas interacções entre os elementos em causa. Desta forma, é essencial que os profissionais desenvolvam uma compreensão mais alargada dos factores que podem proteger os estudantes e dos factores que os podem tornar mais vulneráveis a dificuldades na área do desenvolvimento psicossocial e da realização académica. É neste contexto que, no presente artigo, procuramos compreender o impacto do optimismo disposicional no ajustamento psicológico e rendimento académico demonstrados pelos estudantes do primeiro ano do ensino superior.

Durante a última década, os conceitos de optimismo e de pessimismo têm suscitado muito interesse e investigação nas áreas da psicologia da personalidade, da psicologia social e da psicologia clínica e da saúde (Chang, 1998). De acordo com Sheier e Carver $(1985,1992)$ o optimismo disposicional, definido como uma expectativa generalizada positiva, representa uma variável relativamente estável que promove o bem-estar psicológico e físico. Os indivíduos optimistas são aqueles que, quando confrontados com dificuldades ou adversidades, têm a expectativa de ultrapassar as situações e de alcançar resultados positivos, enquanto que os indivíduos pessimistas face às dificuldades esperam resultados negativos. Estas expectativas de resultados negativos conduzirão a uma tendência marcada para o aparecimento de sentimentos negativos, como a ansiedade, culpa, raiva ou tristeza. Estudos empíricos recentes demonstram que os indivíduos optimistas apresentam níveis melhores de adaptação, quer psicológica quer física, do que os indivíduos pessimistas em diversos contextos (Fontaine \& Seal, 1997; Khoo \& Bishop, 1997; Scheier, Carver, \& Bridges, 2002).

Ao longo dos últimos anos, tem-se tentado compreender o papel que o optimismo desempenha na adaptação física e psicológica dos estudantes à universidade. Estudantes optimistas demonstram menor perturbação psicológica (Aspinwall \& Taylor, 1992; Brissete, Scheier, \& Carver, 2002; Segerstrom, Taylor, Kemeny, \& Fahey, 1998; Stewart et al., 1997), maior bem-estar físico (Aspinwall \& Taylor, 1992) e maior suporte social percebido (Brissete et al., 2002) nesta fase de transição.

No que diz respeito à relação entre o optimismo e o rendimento académico, os resultados não são uniformes. Por exemplo, Schulman (1995) refere que as explicações optimistas para os acontecimentos negativos estão relacionadas com um maior rendimento académico em estudantes universitários e com uma maior produtividade no trabalho. Ao contrário destes resultados, existem estudos que associam os estilos explicativos pessimistas a melhores desempenhos do que os obtidos por estudantes considerados optimistas (Haynes, Ruthig, Perry, Stupnisky, \& Hall, 2006; Ruthig, Perry, Hall, \& Hladkyj, 2004; Satterfield, Monahan, \& Seligman, 1997).

Neste estudo ex post facto prospectivo simples (Montero \& León, 2005), procurámos estudar a relação entre o optimismo disposicional e a sintomatologia psicopatológica, o bem-estar e o rendimento académico demonstrados pelos estudantes que frequentam pela primeira vez o ensino superior. Neste sentido, estabelecemos as seguintes hipóteses: (a) quanto maior for o optimismo disposicional, menor será a sintomatologia psicopatológica demonstrada no final do primeiro semestre; (b) quanto maior for o optimismo disposicional, maior será o bem-estar demonstrado no final do primeiro semestre; (c) há uma relação entre o optimismo disposicional e o rendimento académico demonstrado no final do primeiro semestre.

\section{Método}

\section{Participantes}

Os participantes deste estudo foram 316 estudantes de uma universidade portuguesa, distribuídos por diferentes licenciaturas: licenciaturas de Saúde (42,6\%), licenciaturas de Letras (22,3\%), licenciaturas de Ciências (6,8\%), licenciaturas de Engenharias (9\%) licenciaturas de Economia, Turismo e Gestão e Planeamento em Turismo (10\%) e Design e Administração Pública (9,4\%); sendo que 178 frequentam o ensino universitário $(57,4 \%)$ e 132 frequentam o ensino politécnico (42,6\%). Dos 316 estudantes que participaram neste estudo, 67 são do género masculino $(21,2 \%)$ e 245 são do género feminino $(77,5 \%)$, sendo portanto um grupo maioritariamente feminino. A idade dos participantes varia entre 17 e 38 anos, sendo a média de idades de 18,77 anos, com um desvio-padrão de 2,16. Quanto à residência, 181 (57,3\%) estudantes se mudaram e 123 (42,6\%) permaneceram na residência anterior à entrada no ensino superior, ou seja, a casa dos pais.

\section{Instrumentos}

O optimismo disposicional foi avaliado por meio da versão portuguesa do Life Orientation Test - Revised (LOT-R; Scheier, Carver \& Bridges, 1994) traduzida e adaptada por Monteiro, Tavares e Pereira (2006b). Trata-se de uma escala de auto-resposta com 10 itens com vista a identificar um valor de optimismo disposicional. Três dos itens são cotados de modo inverso e quatro são distractores. Aos respondentes, pede-se que expressem a sua concordância com as afirmações apresentadas, numa escala de tipo likert que vai desde 0 (Discordo totalmente) a 4 (Concordo totalmente). Os estudos psicométricos realizados na versão original (Scheier et al., 1994) revelaram níveis adequados de consistência interna (alfa de Cronbach $=0,78$ ) e de estabilidade temporal.

A sintomatologia psicopatológica foi avaliada através da versão portuguesa do Brief Symptom Inventory (BSI; Derogatis, 1982) traduzida e adaptada por Canavarro (1999). É um inventário de auto-resposta com 53 itens, nos quais o indivíduo deve classificar o grau em que cada problema o afectou durante a 
última semana, numa escala de tipo likert que vai desde 0 (Nunca) a 4 (Muitíssimas vezes). Avalia sintomas psicopatológicos em termos de nove dimensões de sintomatologia (somatização, obsessões compulsões, sensibilidade interpessoal, depressão, ansiedade, hostilidade, ansiedade fóbica, ideação paranóide e psicoticismo) e três índices globais (Índice Geral de Sintomas - IGS -, Total de Sintomas Positivos - TSP - e o Índice de Sintomas Positivos - ISP). Estes últimos são avaliações sumárias de perturbação emocional e representam aspectos diferentes de psicopatologia. Os estudos psicométricos efectuados na versão portuguesa (Canavarro, 1999) revelaram que a escala apresenta níveis adequados de consistência interna para as nove escalas, com valores de alfa de Cronbach entre 0,62 (psicoticismo) e 0,80 (somatização) e coeficientes teste-reteste entre 0,63 (ideacção paranóide) e 0,81 (depressão).

O bem-estar foi avaliado através da versão portuguesa da Échelle de Mesure des Manifestations du Bien-Être Psychologique (EMMBEP; Massé et al., 1998) traduzida e adaptada por Monteiro, Tavares e Pereira (2006a). Trata-se de uma escala de auto-resposta constituída por 25 itens, numa escala de tipo likert de 1 (Nunca) a 5 (Quase sempre), dividida em seis sub-escalas: auto-estima, equilíbrio, envolvimento social, sociabilidade, controlo de si e dos acontecimentos e felicidade. Os estudos psicométricos efectuados na versão original (Massé et al., 1998) revelaram níveis adequados de consistência interna para a nota global (alfa de Cronbach $=0,93$ ) e para as seis subescalas consideradas, com valores de alfa de Cronbach entre 0,71 e 0,85 .

O rendimento académico foi calculado de acordo com a seguinte fórmula: [(número de disciplinas realizadas no primeiro semestre/ número de disciplinas a realizar no primeiro semestre) $\times$ média obtida nas disciplinas realizadas no primeiro semestre]. Incluiu-se na fórmula o número de disciplinas a realizar no primeiro semestre, uma vez que este valor não é igual para todos os cursos incluídos na nossa amostra (por exemplo, no curso de Radiologia, o número de disciplinas a realizar no primeiro semestre é 7, enquanto no curso de Engenharia Mecânica é 5).

\section{Procedimentos}

A selecção dos participantes obedeceu aos seguintes critérios: (a) frequentar o primeiro ano do ensino superior; (b) nunca ter frequentado nenhuma instituição do ensino superior; e, (c) participação voluntária. Após a autorização dos professores, foi efectuado o contacto inicial com os estudantes, momento em que lhes foi explicado o objectivo geral da investigação e se solicitou a sua colaboração para a participação num estudo longitudinal sobre a adaptação ao ensino superior. Os participantes foram informados que iriam completar uma bateria de questionários no início do primeiro semestre, Novembro de 2005, e novamente no final do primeiro semestre, Fevereiro de 2006. Dos 316 estudantes que participaram na coleta de dados no primeiro momento, um total de 140 estudantes ( 35 homens e 105 mulheres) participou também na coleta de dados efectuada no segundo momento. $\mathrm{O}$ optimismo disposicional foi avaliado no primeiro momento e a sintomatologia psicopatológica, o bem-estar e o rendimento académico foram avaliados no segundo momento.

\section{Resultados}

Os resultados que apresentamos de seguida referem-se a três tipos de análises dos dados. Num primeiro momento, apresentamos os resultados referentes à análise da consistência interna (alfa de Cronbach) dos instrumentos de avaliação utilizados no presente estudo. Num segundo momento apresentamos os resultados da análise descritiva das variáveis: sintomatologia psicopatológica, bem-estar e rendimento académico, em função das variáveis demográficas da amostra. Por fim, num terceiro momento apresentamos os resultados da análise correlacional sobre as hipóteses colocadas no início do estudo.

\section{Consistência interna dos instrumentos de avaliação}

A análise de consistência interna do LOT-R indica um valor de alfa de Cronbach adequado $(\alpha=0,74)$, calculado com base nos seis itens incluídos na análise de dados. Os resultados referentes à análise da consistência interna do BSI e da EMMBEP são apresentados nas Tabelas 1 e 2 .

Tabela 1

Número de itens e consistência interna (alfa de Cronbach) do BSI

\begin{tabular}{lcc}
\hline \multicolumn{1}{c}{ Instrumentos de avaliação } & $\begin{array}{c}\text { Número } \\
\text { de itens }\end{array}$ & $\begin{array}{c}\text { Alfa de } \\
\text { Cronbach }\end{array}$ \\
\hline Somatização & 7 & 0,80 \\
Obsessões Compulsões & 6 & 0,81 \\
Sensibilidade Interpessoal & 4 & 0,80 \\
Depressão & 6 & 0,88 \\
Ansiedade & 6 & 0,82 \\
Hostilidade & 5 & 0,79 \\
Ansiedade Fóbica & 5 & 0,74 \\
Ideação Paranóide & 5 & 0,80 \\
Psicoticismo & 5 & 0,74 \\
\hline
\end{tabular}

Por meio da observação da Tabela 1 constatamos que os valores de consistência interna encontrados para o BSI são adequados ou muito adequados, variando entre 0,74 (psicoticismo) e 0,88 (depressão).

Tabela 2

Número de itens e consistência interna (alfa de Cronbach) da EMMBEP

\begin{tabular}{lcc}
\hline \multicolumn{1}{c}{ Instrumentos de avaliação } & $\begin{array}{c}\text { Número } \\
\text { de itens }\end{array}$ & $\begin{array}{c}\text { Alfa de } \\
\text { Cronbach }\end{array}$ \\
\hline Felicidade & 8 & 0,88 \\
Sociabilidade & 4 & 0,82 \\
Controlo de Si e dos Acontecimentos & 3 & 0,82 \\
Envolvimento Social & 3 & 0,65 \\
Auto-Estima & 4 & 0,79 \\
Equilíbrio & 3 & 0,64 \\
\hline
\end{tabular}

A análise dos resultados patentes na Tabela 2 permite-nos referir que os valores de consistência interna encontrados para a EMMBEP são, na sua globalidade, adequados. Com excepção dos valores de alfa de Cronbach encontrados para as sub-escalas envolvimento social $(0,65)$ e equilíbrio $(0,64)$, os restantes são 
muito adequados, variando entre 0,79 (auto-estima) e 0,88 (felicidade).

\section{Caracterização da amostra}

No sentido de explorar a influência das variáveis demográficas: sexo, tipo de ensino (universitário versus politécnico) e mudança de residência na sintomatologia psicopatológica, bem-estar e rendimento académico demonstrados, realizámos testes $t$ de Student para amostras independentes.

No que se refere ao sexo, os resultados demonstraram que não existem diferenças estatisticamente significativas entre os géneros relativamente à sintomatologia psicopatológica, ao bem-estar e ao rendimento académico, com excepção da dimensão ansiedade $[t(136)=-2,15 ; p \leq 0,05]$, sendo que as participantes do sexo feminino demonstraram níveis mais elevados de ansiedade.

No que diz respeito ao tipo de ensino (universitário versus politécnico), os resultados demonstram a existência de diferenças estatisticamente significativas entre os dois grupos relativamente à sintomatologia psicopatológica (ver Tabela 3 ).

Tabela 3

Médias e desvios-padrão de sintomatologia psicopatológica por tipo de ensino (universitário versus politécnico)

\begin{tabular}{|c|c|c|c|c|c|}
\hline Medidas & Ensino Universitário & Ensino Politécnico & $t$ & g.l. & $p$ \\
\hline Somatização & $1,34(0,51)$ & $0,83(2,69)$ & 0,87 & 137 & n.s. \\
\hline Obsessões-Compulsões & $2,24(0,84)$ & $1,28(0,70)$ & 5,73 & 137 & $\leq 0,01$ \\
\hline Sensibilidade Interpessoal & $1,77(0,55)$ & $1,01(0,74)$ & 5,60 & 137 & $\leq 0,01$ \\
\hline Depressão & $1,86(0,73)$ & $1,01(0,77)$ & 4,81 & 137 & $\leq 0,01$ \\
\hline Ansiedade & $1,67(0,56)$ & $0,93(0,66)$ & 4,88 & 136 & $\leq 0,01$ \\
\hline Hostilidade & $1,81(0,71)$ & $0,88(0,63)$ & 6,20 & 136 & $\leq 0,01$ \\
\hline Ansiedade Fóbica & $1,33(0,48)$ & $0,57(0,62)$ & 2,24 & 134 & $\leq 0,01$ \\
\hline Ideação Paranóide & $1,74(0,67)$ & $0,94(0,61)$ & 5,49 & 137 & $\leq 0,01$ \\
\hline Psicoticismo & $1,72(0,60)$ & $0,85(0,67)$ & 5,60 & 137 & $\leq 0,01$ \\
\hline Índice Geral de Sintomas (IGS) & $1,76(0,53)$ & $0,92(0,65)$ & 5,45 & 132 & $\leq 0,01$ \\
\hline
\end{tabular}

Nota: n.s. = não-significativo

A análise dos resultados apresentados na Tabela 3 permite constatar que os estudantes do ensino universitário apresentam níveis mais elevados de sintomatologia psicopatológica do que os estudantes do ensino politécnico e que estas diferenças são estatisticamente significativas para todas as dimensões consideradas (com excepção da somatização) e para o Índice Geral de Sintomas.

Diferenças estatisticamente significativas foram também encontradas entre os dois grupos de tipo de ensino (universitário versus politécnico) em relação ao rendimento académico. Os estudantes do ensino politécnico apresentam níveis mais elevados de rendimento académico quando comparados com os estudantes do ensino universitário e essa diferença é estatisticamente significativa $[t(127)=-3,58 ; p \leq 0,01]$.

Verificámos ainda que não foram encontradas diferenças estatisticamente significativas entre os dois grupos de tipo de ensino (universitário versus politécnico) em relação ao bemestar.

No que diz respeito à influência da mudança de residência, não se verificaram diferenças estatisticamente significativas entre os estudantes que mudaram de residência e os estudantes que permaneceram na residência anterior à entrada no ensino superior relativamente aos diferentes indicadores utilizados para avaliar a adaptação ao ensino superior.

\section{Relação entre o optimismo disposicional e a sintomatologia psicopatológica}

Na Tabela 4, são apresentados os resultados da análise de correlações entre o optimismo disposicional e a sintomatologia psicopatológica. Os resultados descritos mostram a existência de correlações negativas e significativas entre o optimismo e todas as dimensões consideradas (com excepção da somatização) e o Índice Geral de Sintomas. De acordo com o que era esperado, os estudantes que apresentam níveis mais elevados de optimismo disposicional tendem a apresentar menores níveis de sintomatologia psicopatológica no final do primeiro semestre.

Tabela 4

Correlações entre o optimismo disposicional e a sintomatologia psicopatológica

\begin{tabular}{lc}
\hline \multicolumn{1}{c}{ Sintomatologia psicopatológica } & $\begin{array}{c}\text { Optimismo } \\
\text { disposicional }\end{array}$ \\
\hline Somatização & $-0,03$ \\
Obsessões-Compulsões & $-0,38^{* *}$ \\
Sensibilidade Interpessoal & $-0,38^{* *}$ \\
Depressão & $-0,42 * *$ \\
Ansiedade & $-0,37 * *$ \\
Hostilidade & $-0,26 * *$ \\
Ansiedade Fóbica & $-0,37 * *$ \\
Ideação Paranóide & $-0,38^{* *}$ \\
Psicoticismo & $-0,39 * *$ \\
Índice Geral de Sintomas & $-0,37 * *$ \\
\hline$* * p<0,01$ &
\end{tabular}

\section{Relação entre o optimismo disposicional e o bem- estar}

Os resultados da análise de correlações entre o optimismo e o bem-estar são apresentados na Tabela 5. Os resultados descritos mostram a existência de correlações positivas e significativas entre o optimismo e o bem-estar, em todas as dimensões consideradas e o seu indicador global. Mais uma vez, 
de acordo com a hipótese por nós apresentada, os estudantes que apresentam níveis mais elevados de optimismo disposicional tendem a apresentar maiores níveis de bem-estar no final do primeiro semestre.

Tabela 5

Correlações entre o optimismo disposicional e o bem-estar

\begin{tabular}{lc}
\hline \multicolumn{1}{c}{ Bem-estar } & $\begin{array}{c}\text { Optimismo } \\
\text { disposicional }\end{array}$ \\
\hline Felicidade & $0,39 * *$ \\
Sociabilidade & $0,40^{* *}$ \\
Controlo de si e dos acontecimentos & $0,42^{* *}$ \\
Envolvimento & $0,41^{* *}$ \\
Auto-Estima & $0,43^{* *}$ \\
Equilíbrio & $0,19^{*}$ \\
Bem-Estar Global & $0,44^{* *}$ \\
\hline
\end{tabular}

$* p<0,05 \quad * * p<0,01$

\section{Relação entre o optimismo disposicional e o rendimento académico}

Os resultados da análise de correlações entre o optimismo disposicional e o rendimento académico mostram a existência de uma correlação positiva e significativa entre as duas variáveis $(r=0,21 ; p \leq 0,05)$. Isto é, os estudantes que apresentam níveis mais elevados de optimismo disposicional, tendem a apresentar maior rendimento académico no final do primeiro semestre, o que mais uma vez está de acordo com a hipótese formulada que o optimismo disposicional e o rendimento académico estariam relacionados.

\section{Discussão}

Considerados na sua globalidade, os resultados do presente estudo sugerem que o optimismo disposicional é uma variável importante no ajustamento psicológico e rendimento académico demonstrados pelos estudantes que frequentam pela primeira vez o ensino superior.

Em concordância com estudos anteriormente realizados (Aspinwall \& Taylor, 1992; Segerstrom et al, 1998; Stewart et al., 1997), os resultados obtidos sugerem que quanto mais optimistas são os estudantes, maior bem-estar sentem no final do $1^{\mathrm{o}}$ semestre. Relativamente à relação existente entre o optimismo disposicional e a sintomatologia psicopatológica, os dados obtidos são mais uma vez concordantes com o verificado em estudos anteriores (Aspinwall \& Taylor, 1992; Segerstrom et al, 1998; Stewart et al., 1997). Ou seja, quanto mais optimistas são os estudantes, menor sintomatologia psicopatológica apresentam. No que diz respeito à relação entre o optimismo disposicional e o rendimento académico, os nossos resultados sugerem que quanto mais optimistas são os estudantes, melhor é o rendimento académico que apresentam no final do 1o semestre. Apesar deste dado ser consistente com alguns estudos (Brown \& Marshall, 2002; Schulman, 1995), vêm contrariar outros em que a relação observada é a inversa (Haynes et al., 2006; Ruthig et al., 2004; Satterfield et al., 1997).

Merece ainda referência o facto de termos verificado que os estudantes do ensino politécnico apresentam melhor rendimento académico no final do $1^{0}$ semestre do que os estudantes do ensino universitário. Talvez a explicação para este facto resida nas diferenças entre as médias de ingresso das licenciaturas incluídas na nossa amostra. Em virtude do reduzido número de estudantes em algumas das licenciaturas, a análise estatística da relação entre a licenciatura e o rendimento académico tornou-se inviável. De qualquer forma, através da análise pormenorizada das licenciaturas presentes no segundo momento de coleta de dados, pudemos constatar que, na nossa amostra, todas as licenciaturas do ensino politécnico são licenciaturas em Saúde (Enfermagem, Fisioterapia, Gerontologia, etc.), licenciaturas estas com médias de ingresso bastante elevadas. No sentido de compreender melhor esta questão, analisámos as médias de entrada na $1^{\underline{a}}$ fase do ano lectivo 2005/2006 e verificámos que a média mais baixa de ingresso dos cursos do ensino politécnico (150,8 - licenciatura em Gerontologia) é mais elevada do que a média mais alta de ingresso dos cursos do ensino universitário (142,0 - licenciatura em Administração Pública). Uma vez que os resultados escolares obtidos no ensino secundário e/ou em provas de acesso ao ensino superior são apontados como os melhores preditores do desempenho académico no ensino superior (para uma revisão, ver Mathiasen, 1984), a explicação residirá aqui.

Importa ainda tentar compreender os motivos pelos quais os estudantes do ensino universitário apresentaram índices de sintomatologia psicopatológica mais elevados do que os estudantes do ensino politécnico. Uma vez que as médias de ingresso nas licenciaturas de Saúde são bastante mais elevadas do que no cursos do ensino universitário, poderemos supor que os alunos que entraram nas licenciaturas de Saúde, entraram muito provavelmente nas suas primeiras ou segundas opções, o que faz com que se sintam mais realizados e menos vulneráveis ao desenvolvimento de sintomatologia psicopatológica do que a maioria dos demais estudantes.

Cabe ainda salientar o facto de não se verificarem diferenças estatisticamente significativas entre os sexos, relativamente à sintomatologia psicopatológica, ao bem-estar e ao rendimento académico, com excepção da dimensão ansiedade. De acordo com o sugerido por Allen (1999), as mulheres tendem a apresentar níveis mais elevados de desejo de terminar a licenciatura. Por outro lado, o sexo feminino é aquele em que se verificam expectativas mais elevadas relativamente a diferentes dimensões da vida académica, pessoal e sociais associadas ao ensino superior (Soares \& Almeida, 2002). Talvez por estes motivos, sejam as mulheres, nesta fase sentida por muitos estudantes como difícil e exigente, a apresentar maior sintomatologia ansiosa.

Noutra ordem de considerações e apesar dos instrumentos de avaliação LOT-R, BSI e EMMBEP terem sido já alvo de estudos de validade e precisão para a população portuguesa (Canavarro, 1999; Monteiro et al., 2006a, 2006b), torna-se relevante referir o contributo dos resultados aqui apresentados, referentes à análise da consistência interna, para um estudo mais fundamentado das propriedades psicométricas dos mesmos.

As limitações deste estudo deverão ser consideradas. O número de estudantes que participou no segundo momento de coleta de dados, assim como a sua distribuição não equitativa pelas diferentes licenciaturas constituem limitações 
metodológicas que devem ser tidas em conta. Por outro lado, a realização do presente estudo com estudantes apenas de uma universidade portuguesa não permite a generalização dos resultados a nível nacional.

A título de conclusão, podemos dizer que os resultados deste estudo contribuem para aumentar o conhecimento sobre a relação entre o optimismo disposicional e a forma como os estudantes se adaptam ao primeiro ano do ensino superior em Portugal. Intervenções preventivas, promotoras do optimismo, deverão ser delineadas no sentido de facilitar uma transição saudável para a universidade.

\section{Referências}

Allen, D. (1999). Desire to finish college: An empirical link between motivation and persistence. Research in Higher Education, 40, 461-485.

Almeida, L. S. (2001). Acesso, integração e sucesso académico: Uma análise reportada aos estudantes do $1^{\circ}$ ano. In R. B. Sousa, E. Sousa, F. Lemos \& C. Januário (Orgs.), III Simpósio - Pedagogia na Universidade (pp. 223-240). Lisboa: Universidade Técnica de Lisboa.

Aspinwall, L. G., \& Taylor, S. E. (1992). Modeling cognitive adaptation: A longitudinal investigation of the impact of individual differences and coping on college adjustment and performance. Journal of Personality and Social Psychology, 63, 989-1003.

Brissette, I., Scheier, M. F., \& Carver, C. S. (2002). The role of optimism in social network development, coping, and psychological adjustment during a life transition. Journal of Personality and Social Psychology, 82, 102-111.

Brown, J. D., \& Marshall, M. A. (2002). Great expectations: Optimism and pessimism in achievement settings. In C. Chang (Org.), Optimism and pessimism: Implications for theory, research, and practice (pp. 239-255). Washington, DC: American Psychological Association.

Canavarro, M. C. (1999). Inventário de Sintomas Psicopatológicos - B.S.I.. In M. R. Simões, M. M. Gonçalves, \& L. S. Almeida (Orgs.), Testes e provas psicológicas em Portugal (vol. 2, pp. 95-109). Braga: APPORT/SHO.

Chang, E. C. (1998). Does dispositional optimism moderate the relation between perceived stress and psychological well-being: A preliminary investigation. Personality and Individual Differences, 25, 233-240.

Cunha, S. M., \& Carrilho, D. M. (2005). O processo de adaptação ao ensino superior e o rendimento académico. Psicologia Escolar e Educacional, 9(2), 215-224.

Derogatis, L. R. (1982). Self-report measures of stress. In L. Goldberger \& S. Brenznitz (Orgs.), Handbook of stress (pp. 270-294). Nova York: Free Press.

Diniz, A. M., \& Almeida, L. S. (2005). Escala de Integração Social no Ensino Superior (EISES): metodologia de construção e validação. Análise Psicológica, 23(4), 461-476.

Diniz, A. M., \& Almeida, L. S. (2006). Adaptação à universidade em estudantes do primeiro ano: estudo diacrónico da interacção entre o relacionamento com pares, o bem-estar pessoal e o equilíbrio emocional. Análise Psicológica, 24(1), 29-38

Fernandes, E. M., Maia, A., Meireles, C., Rios, S., Silva, D., \& Feixas, G. (2005) Dilemas implicativos e ajustamento psicológico: um estudo com alunos recém-chegados à Universidade do Minho. International Journal of Clinical and Health Psychology, 5, 285-304

Ferraz, M. F., \& Pereira, A. S. (2002). A dinâmica da personalidade e o homesickness (saudades de casa) dos jovens estudantes universitários. Psicologia, Saúde \& Doenças, 3(2), 149-164.

Fontaine, K. R., \& Seal, A. (1997). Optimism, social support, and premenstrual dysphoria. Journal of Clinical Psychology, 53, 234-247.

Haynes, T. L., Ruthig, J. C., Perry, R. P., Stupnisky, R. H., \& Hall, N. C. (2006). Reducing the academic risks of over-optimism: The longitudinal effects of attributional retraining on cognition and achievement. Research in Higher Education, 47(7), 755- 779

Khoo, S., \& Bishop, G. D. (1997). Stress and optimism: Relationships to coping and well-being. Psychologia: An International Journal of Psychology in the Orient, 40, 29-40

Machado, C., \& Almeida, L. S. (2001). Vivências académicas: Análise diferencial em estudantes dos $1^{\circ}$ e $4^{\stackrel{o}{a}}$ anos do Ensino Superior. In J. Tavares \& R. Santiago (Orgs.), Ensino superior. (In)sucesso académico (pp. 133-145). Porto: Porto Editora.

McLaughlin, G. W., Brozovsky, P. V., \& McLaughlin, J. S. (1998). Changing perspectives on student retention: A role for institutional research. Research in Higher Education, 39(1), 1-17.

Massé, R., Poulin, C., Dassa, C., Lambert, J., Bélair, S., \& Battaglini, A. (1998). Élaboration et validation d'un outil de mesure du bien-être psychologique : L'É.M.M.B.E.P. Revue Canadienne de Santé Publique, 89(5), 352-357.

Mathiasen, R. E. (1984). Predicting college academic achievement: A research review. College Student Journal, 18, 380-386.

Mercuri, E., \& Polydoro, S. A. J. (Orgs.). (2003). Estudante universitário. características e experiências de formação. Taubaté, SP: Cabral Editora.

Monteiro, S., Tavares, J., \& Pereira, A. (2006a). Estudo das características psicométricas da escala de medida de manifestação de bem-estar [Texto completo]. In I. Leal, J. L. Ribeiro \& S. N. Jesus (Orgs.), Actas do $6^{o}$ Congresso Nacional de Psicologia da Saúde, Bem-Estar e Qualidade de Vida (pp. 53-58). Lisboa: ISPA Edições.

Monteiro, S., Tavares, J., \& Pereira, A. (2006b). Portuguese study of Life Orientation Test - Revised (LOT-R) [Resumo]. In A. Maia, C. Sousa, C. Fonte, E. Fernandes, G. Rodrigues, P. B. Albuquerque, \& T. Freire (Org.), 3rd European Conference on Positive Psychology. Book of Abstracts (p. 91). Braga: Universidade do Minho.

Montero, I., \& León, O. G. (2005). Sistema de clasificación del método en los informes de investigación en Psicología. International Journal of Clinical and Health Psychology, 5, 115-127.

Nico, J. B. (2000). Tornar-se estudante universitário(a): Contributo do conforto académico na definição de uma estratégia curricular de sucesso. Tese de doutorado não-publicada, Universidade de Évora, Évora, Portugal.

Pantages, T. J., \& Creedon, C. F. (1978). Studies of college attrition: 1950-1975. Review of Educational Research, 48(1), 49-101.

Polydoro, S. A. J., Primi, R., Serpa, M. N., Zaroni, M. M. H., \& Pombal, K. C. P. (2001). Desenvolvimento de uma escala de integração ao ensino superior. Psico-USF, 6(1), 11-17

Ruthig, J. C., Perry, R. P., Hall, N. C., \& Hladkyj, S. (2004). Optimism and attributional retraining: Longitudinal effects on academic achievement, test anxiety, and voluntary course withdrawal in college students. Journal of Applied Social Psychology, 34, 709-730.

Santos, L., \& Almeida, L. S. (2001). Vivências académicas e rendimento escolar: estudo com alunos universitários do $1^{\circ}$ ano. Análise Psicológica, 19(2), 205-217.

Satterfield, J. M., Monahan, J., \& Seligman, M. E. P. (1997). Law school performance predicted by explanatory style. Behavioral Sciences and the Law, 15, 95-105.

Scheier, M. F., \& Carver, C. S. (1985). Optimism, coping and health: Assessment and implications of generalized outcome expectancies. Health Psychology, 4, 219-247.

Scheier, M. F., \& Carver, C. S. (1992). Effects of optimism on psychological and physical well-being: Theoretical overview and empirical update. Cognitive Therapy and Research, 16, 201-228.

Scheier, M. F., Carver, C. S., \& Bridges, M. W. (1994). Distinguishing optimism from neuroticism (and trait anxiety, self-mastery, and self-esteem): A reevaluation of the Life Orientation Test. Journal of Personality and Social Psychology, 67, 1063-1078.

Scheier, M. F., Carver, C. S., \& Bridges, M. W. (2002). Optimism, pessimism, and psychological well-being. In C. Chang (Org.), Optimism and pessimism: Implications for theory, research, and practice (pp. 189-216). Washington: 
American Psychological Association.

Schleich, A. L. R., Polydoro, S. A. J., \& Santos, A. A. A. (2006). Escala de satisfação com a experiência académica de estudantes do ensino superior. Avaliação Psicológica, 5(1), 11-20.

Schulman, P. (1995). Explanatory style and achievement in school and work. In G. M. Buchanan \& M. E. P. Seligman (Orgs.), Explanatory style (pp. 159-171). Hillsdale: Lawrence Erlbaum.

Segerstrom, S. C., Taylor, S. E., Kemeny, M. E., \& Fahey, J. L. (1998). Optimism is associated with mood, coping, and immune change in response to stress. Journal of Personality and Social Psychology, 74, 1646-1655.

Soares, A. P. (2003). Transição e adaptação ao Ensino Superior: Construção e validação de um modelo multidimensional de ajustamento de jovens ao contexto universitário. Tese de doutorado não-publicada, Universidade do Minho, Braga, Portugal.

Soares, A. P., \& Almeida, L. S. (2002). Trajectórias escolares e expectativas académicas dos candidatos ao ensino superior: contributos para a definição dos alunos que entraram na Universidade do Minho. Braga: Universidade do Minho.

Stewart, S. M., Betson, C., Lam, T. H., Marshall, I. B., Lee, P. W., \& Wong, C. M. (1997). Predicting stress in first year medical students: A longitudinal study. Medical Education, 3, 163-168.

Tavares, J., Santiago, R. A., \& Lencastre, L. (1998). Insucesso no $1^{\underline{o}}$ ano do ensino superior: um estudo no âmbito dos cursos de licenciatura em ciências e engenharia na Universidade de Aveiro. Aveiro: Universidade de Aveiro.

Tinto, V. (1993). Leaving college: Rethinking the causes and cures of student attrition (2a ed.). Chicago: University of Chicago Press.

Vendramini, C. M. M., Santos, A. A. A., Polydoro, S. A. J., Sbardelini, E. T. B., Serpa, M. N. F., \& Natário, E. G. (2004). Construção e validação de uma escala sobre avaliação da vida académica (EAVA). Estudos de Psicologia, $9(2), 259-268$.

Zitzow, D. (1984). The College Adjustment Rating Scale. Journal of College Student Personnel, 25, 160-164.

Sara Otília Marques Monteiro, mestre em Psicologia pela Universidade de Lisboa, é doutoranda em Psicologia na Universidade de Aveiro (Portugal). Endereço para correspondência: Departamento de Ciências da Educação, Universidade de Aveiro, Campus Universitário de Santiago, 3810-193, Aveiro, Portugal. Tel.: 00351234370 353.Fax: 00351234370 640.E-mail: smonteiro@ua.pt José Pereira da Costa Tavares, doutor em Filosofia pela Universidade Católica de Louvain (Bélgica), é professor catedrático na Universidade de Aveiro (Portugal).E-mail: jtav@ua.pt Anabela Maria de Sousa Pereira, doutora em Psicologia pela University of Hull (Reino Unido), é professora auxiliar (Psicologia da Saúde) na Universidade de Aveiro (Portugal). E-mail: anabelapereira@ua.pt 